\title{
Symmetry-breaking assembled porous calcite microspheres and their multiple dental applications
}

\author{
Ming $\mathrm{Ma}^{1 \dagger}$, Yanhong $\operatorname{Yan}^{2 \dagger}$, Chao Qi ${ }^{1}$, Shengcai Qi ${ }^{3}$, Shixiong Chern ${ }^{1}$, Guangwei Shang ${ }^{3}$, \\ Raorao Wang ${ }^{3 *}$ and Hangrong Chen ${ }^{1 *}$
}

\begin{abstract}
Biomedical applications of porous calcium carbonate $\left(\mathrm{CaCO}_{3}\right)$ microspheres have been mainly restricted by their aqueous instability and low remineralization rate. To overcome these obstacles, a novel symmetry-breaking assembled porous calcite microsphere (PCMS) was constructed in an ethanol/water mixed system using a two-step vapor-diffusion/aging crystallization strategy. In contrast to the conventional additive-induced crystallization method, the present strategy was performed under mild conditions and was free from any foreign additives, thus avoiding the potential contamination of the final product. Meanwhile, the prepared PCMSs were characterized by their highly uniform spherical morphology and large open pores, which are favorable for large protein delivery. An antimicrobial study of immunoglobulin Y (IgY)-loaded PCMSs revealed excellent antimicrobial activity against Streptococcus mutans. More importantly, they showed surprisingly rapid transformation to bone minerals in physiological medium. Evaluation of the in vitro efficacy of PCMSs in dentinal tubule occlusion demonstrated their powerful potential to serve as a catalyst in the repair of dental hard tissue. Therefore, the developed PCMSs show great promise as multifunctional biomaterials for dental treatment applications.
\end{abstract}

Keywords: porous calcium carbonate, remineralization, dentinal tubule occlusion, antimicrobial, protein delivery

\section{INTRODUCTION}

In dental materials research, the construction of inorganic materials with novel structures and high bone activity using facile and environmentally friendly preparation processes has become an important area. Among the inorganic materials being explored in recent years, $\mathrm{CaCO}_{3}$, as one of the main biomineral components produced naturally by organisms, is very suitable for dental treatment because of its excellent biodegradability and biocompatibility [1-3]. For example, $\mathrm{CaCO}_{3}$ particles have been applied widely as effective desensitizing agents, abrasives, and slow-release agents in the cleaning and repair of dental hard tissue [2,4-6]. In particular, $\mathrm{CaCO}_{3}$ microparticles (MPs), with unique porous and high surface area characteristics, show promise because of their significant advantage in loading and controlled release of other guest substances, such as drugs and proteins [7]. Thus, the crystallization and morphological control of $\mathrm{CaCO}_{3}$ as a porous structure have attracted extensive research attention in recent years [8-11].

A number of studies to establish porous $\mathrm{CaCO}_{3} \mathrm{MPs}$ have been carried out under diverse conditions [12-15]. Typically, the vaterite polymorph of $\mathrm{CaCO}_{3}$, with its micro-spherical and porous structure, can be fabricated simply via the conventional process reported by Volodkin et al. [16]. However, since vaterite is rather unstable in a humid environment, this porous $\mathrm{CaCO}_{3}$ will easily convert to non-porous rhombohedral calcite overnight [16]. Recently, several modification approaches, such as layer-by-layer electrostatic adsorption, double hydrophilic block copolymers coating, and nanodots embedding, have been attempted to stabilize vaterite MPs [14,17-19]. The above foreign impurities can inhibit the nucleation of calcite from metastable vaterite phase. Unfortunately,

${ }^{1}$ State Key Lab of High Performance Ceramics and Superfine Microstructures, Shanghai Institute of Ceramics, Chinese Academy of Sciences, Shanghai 200050, China

${ }^{2}$ Shanghai Engineering Research Center of Tooth Restoration and Regeneration, Department of Pediatric Dentistry, School of Stomatology, Tongji University, Shanghai 200072, China

${ }^{3}$ Department of Stomatology, Shanghai Tenth People's Hospital, Tongji University, Shanghai 200072, China

† These authors contributed equally to this work.

*Corresponding authors (emails: raoraowang@tongji.edu.cn (Wang R); hrchen@mail.sic.ac.cn (Chen H)) 
these strategies generally suffer from complex synthetic pathways and potential additives-induced toxicity, which restrict their practical biomedical applications.

Calcite is the most attractive phase for the construction of porous $\mathrm{CaCO}_{3} \mathrm{MPs}$, because they are more structurally stable than their vaterite counterparts $[20,21]$. It is believed that the porous calcite MPs with high surface area could provide multiple new possibilities for medical and dental applications. However, it is a challenge to introduce porosity into calcite MPs because of their uncontrollable crystallization kinetic behavior. One alternative that has been developed is the porous mesocrystals of calcite with peanut-like morphology, which are fabricated from a $\mathrm{CO}_{2}-\mathrm{H}_{2} \mathrm{O}-\mathrm{Ca}(\mathrm{OH})_{2}$ slurry [22]. Unfortunately, organic additives are still required in this synthetic route to generate the pore structure in the calcite product. Moreover, this particle has a rather small pore size, which greatly limits its application for bio-macromolecule delivery $[23,24]$. Therefore, developing an additive-free synthetic strategy to prepare porous $\mathrm{CaCO}_{3}$ MPs in the stable calcite form that have large enough pore channels remains a challenge.

In this study, we focused on the design of a new generation of $\mathrm{CaCO}_{3}$, i.e., pure porous calcite microspheres (PCMSs), using a two-step vapor-diffusion/aging crystallization strategy, which may fulfill all the above-mentioned demands. In contrast to the conventional additive-induced crystallization method, the structural manipulation for the desired morphology of PCMSs is based on the inhibitory effect of ethanol on the symmetrical growth of $\mathrm{CaCO}_{3}$. Importantly, the structural stability of the newly designed PCMSs in phosphate-free water solution was maintained for more than three months, which was not a feature of other previously reported porous $\mathrm{CaCO}_{3} \mathrm{MPs}$. Meanwhile, the unique large-pore structure endows PCMSs with high adsorption capacity for antibacterial protein drug, resulting in an efficient antibacterial activity against harmful oral bacteria. Moreover, the performances of PCMSs in remineralization and treating exposed dentin were also investigated.

\section{EXPERIMENTAL SECTION}

\section{Materials}

Calcium chloride $\left(\mathrm{CaCl}_{2}\right)$, calcium chloride dihydrate $\left(\mathrm{CaCl}_{2} \cdot 2 \mathrm{H}_{2} \mathrm{O}\right)$, sodium carbonate $\left(\mathrm{Na}_{2} \mathrm{CO}_{3}\right)$, ammonium bicarbonate $\left(\mathrm{NH}_{4} \mathrm{HCO}_{3}\right)$, and ethanol were purchased from Aldrich Sigma and used without further purification. Immunoglobulin Y (IgY) powder was obtained from Equitech-Bio. Teeth samples were obtained from healthy human subjects (20-40 years old) after approval by Shanghai Tenth People's Hospital.

\section{Physical characterization}

Scanning electron microscopy (SEM) images were obtained using two different microscopes: a Tecnai G2 F20 and a HITACHI S-4800. Transmission electron microscopy (TEM) images were acquired using a JEM-2100F electron microscope with an accelerating voltage of $200 \mathrm{kV}$. The TEM size measurement was based on ImageJ $1.40 \mathrm{G}$ software. The UV-vis spectra were acquired on a Shimadzu UV-3101PC UV-vis absorption spectrophotometer. The nitrogen adsorption-desorption curves were obtained on a Micrometitics Tristar 3000 system. The Brunauer-Emmett-Teller (BET) method was used to calculate the specific surface area. Meanwhile, the corresponding pore size distribution was measured by the Barrett-Joyner-Halenda (BJH) method. The Fourier-transform infrared (FTIR) spectroscopy analyses were carried out on a Nicolet 7000-C spectrometer. X-ray diffraction (XRD) diffraction patterns were recorded on a Rigaku $\mathrm{D} / \mathrm{Max}-2200 \mathrm{PC} \mathrm{X}$-ray diffractometer using $\mathrm{Cu} \mathrm{Ka}$ radiation $(40 \mathrm{kV}$ and $40 \mathrm{~mA})$ with a scanning rate of $4^{\circ} \mathrm{min}^{-1}$.

\section{Preparation of $\mathrm{CaCO}_{3} \mathrm{MPs}$}

The preparation procedure of PCMSs was divided into two steps: self-assembly of primary calcite MPs in an ethanolbased precursor solution and a subsequent aging crystallization process.

In the first step, $400 \mathrm{~mL}$ of ethanol with $\mathrm{CaCl}_{2} \cdot 2 \mathrm{H}_{2} \mathrm{O}$ $(10 \mathrm{~g})$ were transferred into a broad-necked flask and then covered with plastic film containing several pores. The broad-necked flask was put into a desiccator along with a bottle of $\mathrm{NH}_{4} \mathrm{HCO}_{3}$ (about $100 \mathrm{~g}$ ). The desiccator was sealed and left at $30^{\circ} \mathrm{C}$ for 7 days. The color of precursor solution changed to milky white during this process. The white precipitate was collected by centrifugation $(10,000$ $\mathrm{rpm}$ ) and washed with ethanol (total volume: $100 \mathrm{~mL}$ ). In addition, to understand the effect of the water/ethanol ratio $R$ ( $R$ represents the volume ratio of water to ethanol) in the precursor solution on the phase selection and morphological change, an anhydrous $\mathrm{CaCl}_{2}\left(0.17 \mathrm{molL}^{-1}\right)$-containing ethanol solution $(R=0)$ and $\mathrm{CaCl}_{2} \cdot 2 \mathrm{H}_{2} \mathrm{O}\left(0.17 \mathrm{~mol} \mathrm{~L}^{-1}\right)$-containing water/ethanol mixed solution $(R=1 / 40)$ were also chosen as precursor solutions for comparison.

In the second step, the freeze-dried products obtained in the first step (about $800 \mathrm{mg}$ ) were immersed in a mixed water/ethanol solution $(R=1 / 50)$ at $30^{\circ} \mathrm{C}$ for 7 days. Finally, the white precipitate was collected by centrifugation 
$(10,000 \mathrm{rpm})$ and freeze-dried overnight for further use.

\section{Structural stability of PCMSs}

To study the structural stability of the PCMSs in water solution, $10 \mathrm{mg}$ of freeze-dried PCMSs were soaked in $15 \mathrm{~mL}$ of deionized water and kept in static conditions at room temperature for three months. The sample was collected by centrifugation $(10,000 \mathrm{rpm})$ for further characterizations.

\section{IgY protein loading process}

The PCMS powdered sample $(60 \mathrm{mg})$ was mixed with deionized water solution $(7.5 \mathrm{~mL})$ containing IgY protein at an initial concentration of $1 \mathrm{mg} \mathrm{mL}^{-1}$ by a gentle stirring process at $4^{\circ} \mathrm{C}$. The sample was collected after centrifugation at 10,000 rpm and washed with water once. The sample was then freeze-dried and stored for further use. The content of IgY loaded in the carriers was determined by UV-vis spectroscopy. Briefly, the supernatant IgY solution was collected by centrifugation $(10,000 \mathrm{rpm})$. The concentration of $\operatorname{IgY}$ in the supernatant was measured by UV-vis spectroscopy at a wavelength of $278 \mathrm{~nm}$. The IgY protein loading capacity was calculated using the following equation: IgY loading capacity $(\%)=100 \times$ (initial mass of IgY - mass of IgY in the supernatant) / (mass of PCMSs + initial mass of IgY - mass of IgY in the supernatant).

\section{IgY protein release behavior}

The PCMSs-IgY (25 mg) was added into a plastic tube containing $12 \mathrm{~mL}$ phosphate buffered saline (PBS) solution. Then, the tube was placed in a shaking table with $40 \mathrm{rpm}$ at $37^{\circ} \mathrm{C}$. At different time intervals, the supernatants were collected by centrifugation $(14,000 \mathrm{rpm})$ and the concentrations of the released IgY were measured using UV-vis spectroscopy.

\section{Antibacterial property}

The inhibitory effects of single PCMSs, free IgY, and PCMSs-IgY were examined against Streptococcus mutans (S. mutans) using a colony counting approach. The IgY concentrations of the free IgY group and PCMSs-IgY group were fixed at $4.5 \mathrm{mg} \mathrm{mL}^{-1}$. In brief, the $S$. mutans suspension (about $10^{5}$ colony-forming units $(\mathrm{CFU}) / \mathrm{mL}$ ) was firstly mixed with each sample in a centrifuge tube for $30 \mathrm{~s}$ using a vortex mixer. Then, the tubes were incubated at $37^{\circ} \mathrm{C}$ under anaerobic conditions for $12 \mathrm{~h}$. Afterwards, the diluted $S$. mutans suspensions were spread on an agar plate and further incubated for $48 \mathrm{~h}$ anaerobically. Finally, the CFU on the agar plate was counted for each group.

\section{In vitro study of the transformation of PCMSs into bone minerals}

To study the structural transformation of PCMSs to apatite, $20 \mathrm{mg}$ of freeze-dried PCMSs were immersed in $15 \mathrm{~mL}$ of PBS solution (1×, pH 7.4) and filtered human saliva, separately. The PCMSs suspensions were kept in static conditions at $37.5^{\circ} \mathrm{C}$ for different periods $(2$ and $24 \mathrm{~h}$ ). In addition, for a comparative study, a typical rhombohedral calcite microcrystal was also fabricated by mixing $0.33 \mathrm{~mol} \mathrm{~L}^{-1} \mathrm{Na}_{2} \mathrm{CO}_{3}$ and $0.033 \mathrm{molL}^{-1} \mathrm{CaCl}_{2}$ in deionized water at $25^{\circ} \mathrm{C}$ for $24 \mathrm{~h}$.

\section{Evaluation of the dentin tubules occlusion}

Dentin discs with a thickness of about $2 \mathrm{~mm}$ were cut from intact human molars. The dentin discs were then polished using a polishing wheel to obtain an even dentin surface. To open the dentin tubules, the dentin discs were immersed in a $1 \%$ citric acid solution for $30 \mathrm{~min}$, followed by ultrasonic washing in deionized water for $10 \mathrm{~min}$.

The PCMSs paste was prepared by suspending PCMSs $\left(50 \mathrm{mg} \mathrm{mL}^{-1}\right)$ in a glycerinum solution. The dentin specimen was wetted using PBS solution. Then, $200 \mu \mathrm{L}$ of the above PCMSs paste was applied on the entire surface of each dentin specimen by brushing with a small toothbrush for $2 \mathrm{~min}$. The treated dentin specimens were incubated in $30 \mathrm{~mL}$ of PBS solution and shaken at $80 \mathrm{rpm}$ at $37^{\circ} \mathrm{C}$. After $15 \mathrm{~min}$ of incubation, the samples were rinsed gently using deionized water to remove the excess products from the dentin surface. The washed samples were then allowed to sit in $\mathrm{PBS}$ solution at $37^{\circ} \mathrm{C}$. The dentin discs were brushed twice each day (morning and night) for 7 days. For comparison, a commercial $\mathrm{CaCO}_{3}$ in toothpaste (Micro-chalk $\mathrm{CaCO}_{3}$ powders supplied from Omya International, denoted as MCCPs) was also used to treat dentin samples based on the above procedure. All the dentin specimens were freeze-dried before SEM observations.

\section{RESULTS AND DISCUSSION}

\section{Synthetic route and structural characterizations of PCMSs}

As shown in Fig. 1a, the PCMSs were fabricated in two steps: self-assembly of a primary calcite MPs and a subsequent aging crystallization process. In the first step, ammonia and carbon dioxide were reacted with $\mathrm{CaCl}_{2}$ in a precursor solution with a ratio of water to ethanol of $R=1 / 160$ by a vapor-diffusion process lasting for 7 days. Spherical MPs (denoted as SMP1s) with rough surfaces were observed under SEM analysis (Fig. S1a). Meanwhile, the high-magnification SEM image (Fig. S1b) of SMP1s indicates that the spherical structure comprised a great number of $3-5 \mathrm{~nm}$ 

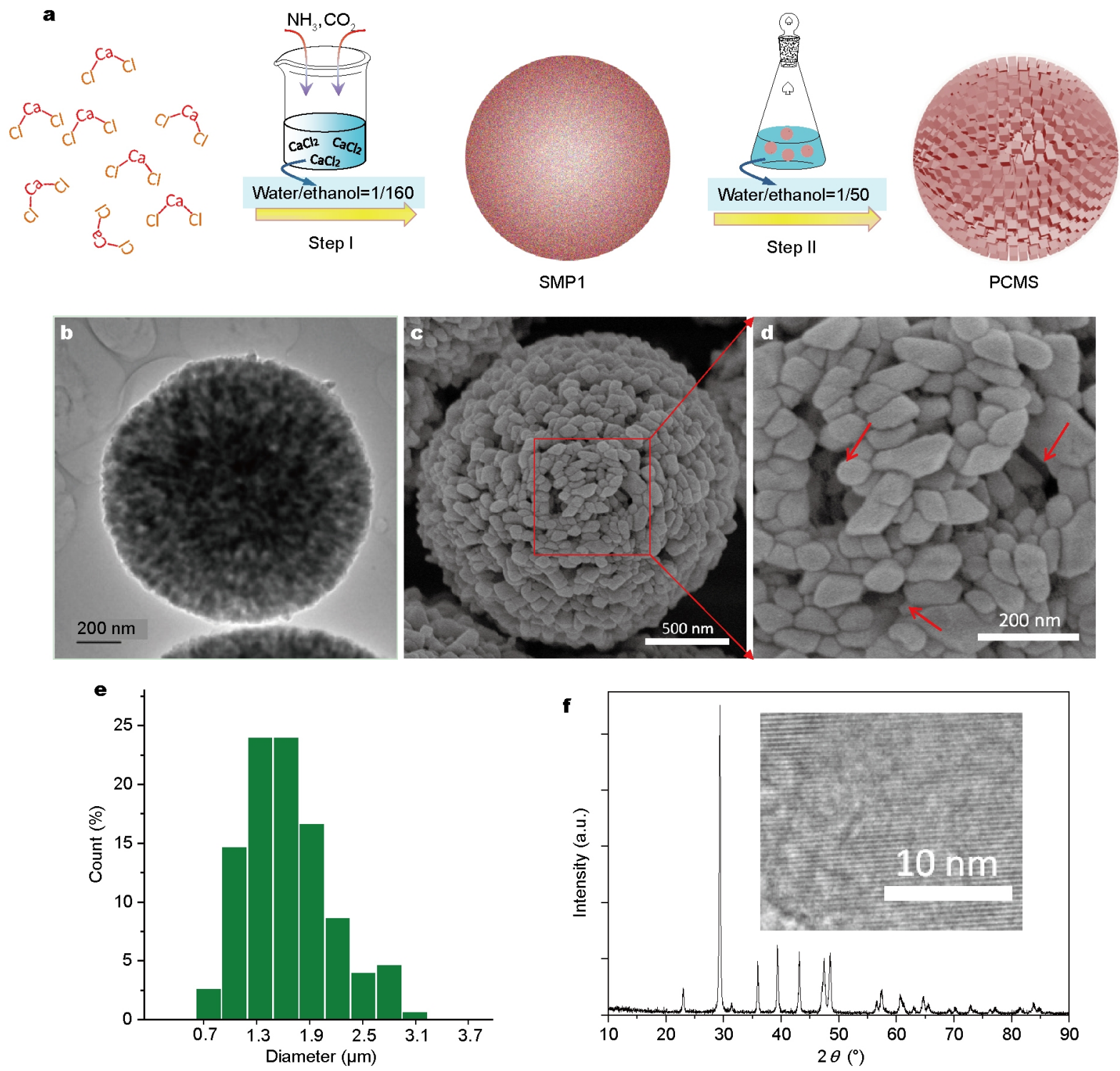

Figure 1 (a) Schematic illustration of the two-step synthetic procedure for PCMSs. Step I: vapor-diffusion process. Step 2: aging crystallization process via soaking PCMS in a mixed water/ethanol solution $(R=1 / 50)$ at $30^{\circ} \mathrm{C}$ for 7 days. (b-e) Morphological and XRD characterizations of PCMSs: (b) TEM image of PCMSs. (c, d) SEM images of PCMSs. Fig. 1d shows the enlargement of the selected square region in (c). The arrows indicate the large-pore on the MPs surface. (e) TEM size distribution of PCMSs. Over 200 particles in different fields of views were analyzed for the size distribution. (f) The XRD pattern of PCMSs. The inset high-resolution TEM image shows the lattice fringe of PCMSs.

carbonate nanodots (NDs) with a close stacking architecture, suggesting the existence of amorphous $\mathrm{CaCO}_{3}$ in SMP1. The XRD patterns (Fig. S2) of the $\mathrm{CaCO}_{3}$ precipitates collected at different intervals (day 1, 4 and 7) during the vapor-diffusion process indicate that the crystallization degree of the calcite phase was gradually enhanced with time. Thus, a considerable proportion of the amorphous $\mathrm{CaCO}_{3}$ was converted readily into calcite phase because of the presence of water. It is noted that the obtained SMP1s after 7 days of action still showed a relatively broad XRD peak (Fig. S2), revealing their low crystalline nature. In previous reports, the typical rhombohedral calcite could be formed by the gas-diffusion approach in a pure water system [25]. In comparison, the formation of lowly crystallized and spherical calcite MPs in the present strategy indicates strongly that a different crystal growth pathway using ethanol as dominant solvent had occurred. It is reasonable to believe that the presence of ethanol could greatly reduce the solubility of the amorphous $\mathrm{CaCO}_{3}$ nano-precipitations at the early stage of the reaction, thus 
retarding the diffusion of their ions and promoting symmetric growth into the most stable rhombohedral calcite [26].

In the second step, the SMP1s were aged by soaking the samples in a mixed water/ethanol solution $(R=1 / 50)$ at $30^{\circ} \mathrm{C}$ for 7 days. Surprisingly, the spherical shaped SMP1s changed into a well-defined inter-connective porous structure upon aging, as shown in Fig. 1b-d and Fig. S3. The particle size of the PCMSs was in the range of 0.7 to $3.0 \mu \mathrm{m}$, (average $1.8 \mu \mathrm{m}$ as assessed by statistical analysis) (Fig. 1e). Furthermore, the nitrogen-sorption isotherm (Fig. S4) shows a major capillary condensation step in the relative pressure range of $0.90-0.95$, implying that PCMSs possess macroporous structure. BET analysis revealed a high surface area of $24 \mathrm{~m}^{2} \mathrm{~g}^{-1}$ and a pore size distribution of about 20 to $80 \mathrm{~nm}$. As shown in Fig. 1d, the porous structure originated mainly from the void space of symmetry-breaking aggregations among a great number of small sized rhombohedral blocks. To clarify the identity of these building blocks, we further conducted a crystal structure analysis of the PCMSs. The significantly sharp diffraction peaks of calcite and the corresponding highly ordered lattice fringes in a representative building block were observed on the XRD pattern and high-resolution SEM images (Fig. 1f), respectively, demonstrating that the crystallization degree of SMP1s had been increased significantly after the second-step aging process. More significantly, the unique porous calcite structure of the final PCMSs was constructed, for the first time, using of an additive-free strategy.

For practical use in biomedical applications, including toothpastes, cosmetics, protein encapsulation, and biosensing, the structural stability of $\mathrm{CaCO}_{3}$ in aqueous solutions is highly desired [7,27-29]. Previous research showed that porous vaterite samples in the absence of stabilizing additives would convert easily to non-porous rhombohedral calcite under ambient conditions [14]. In contrast, the typical structural features of our PCMSs, including their spherical shape, porous structure, and high-crystallinity, were maintained well after being soaked in deionized water for three months (Fig. S5). Such high-stability performance of the PCMSs was attributed to the extremely low thermodynamic energy of their inherent calcite phase. Importantly, this phenomenon is significant for the practical application of porous $\mathrm{CaCO}_{3}$, since further stabilization route by adding organic impurities can be completely avoided.

Structural discrimination in water/ethanol binary solution Three different specific water/ethanol ratios of the binary solution were selected to check the influence of water on the structural discrimination of the final $\mathrm{CaCO}_{3}$ products. The SEM image in Fig. 2a demonstrates that the raspberry-like nanoparticles (NPs) with a uniform particle size of about $110 \mathrm{~nm}$ (denoted as RNP1s) were obtained in the first step without adding the water component to the solvent. The amorphous phase of NPs, as confirmed by the XRD diffraction pattern shown in Fig. S6a, indicates that the crystallization process of $\mathrm{CaCO}_{3}$ hardly occurred in the absence of water. The structural stability of amorphous $\mathrm{CaCO}_{3}$ could be ascribed to the blocking effect of ethanol on the particle surface as the transports of $\mathrm{Ca}^{2+}$ and $\mathrm{CO}_{3}{ }^{2-}$ for crystallization are strongly hindered [30]. Furthermore, after the aging process in the second step, spherical shaped vaterite NPs of about $700 \mathrm{~nm}$ (denoted as VNPs) were obtained, comprising small substructures with a similar size to the RNP1s, as seen from the SEM image (Fig. 2b) and XRD analysis (Fig. S6b). Thus, the growth of the VNPs during the aging process seemed to occur by the combined actions of the random aggregation of amorphous RNP1s and a vaterite-oriented crystallization process.

Furthermore, with an increase of the $R$-value to $1 / 160$ in the first step (by replacing $\mathrm{CaCl}_{2}$ with its hydrated counterpart $\mathrm{CaCl}_{2} \cdot 2 \mathrm{H}_{2} \mathrm{O}$ ), SMP1s with non-porous and spherical morphologies were obtained in the first step of reaction. Compared with the RNP1s obtained in the water-free system, the SMP1s showed significantly larger particle sizes (Fig. 2c). Based on this result, we speculated that the water molecules in the reaction system might be involved in bridging the hydrogen bond networks across the interfaces of the primary $\mathrm{CaCO}_{3} \mathrm{NDs}$, thus leading to the formation of micrometer-sized aggregates. Furthermore, after the aging process, the observed rhombohedral building blocks of the PCMSs were significantly larger than that of the carbonate NDs of the SMP1s (Fig. 2c, d), revealing that the primary NDs have the tendency to decrease both their bulk lattice energy and surface energy by inter-particle fusion and subsequent crystallization [31]. Considering the co-existence of amorphous $\mathrm{CaCO}_{3}$ and calcite in SMP1s, as noted above, the growth of rhombohedral building blocks in our PCMSs seemed to have occurred by the oriented fusion of amorphous $\mathrm{CaCO}_{3}$ units onto the adjacent calcite surface because of the relatively stronger dipolar field for calcite phase. For this reason, it is easily understood that the local void space can be formed after the amorphous $\mathrm{CaCO}_{3}$ molecules moved away from their original position, thus leading to the formation of porous structure in PCMSs.

With a further increase of the $R$-value to $1 / 40$, two kinds 


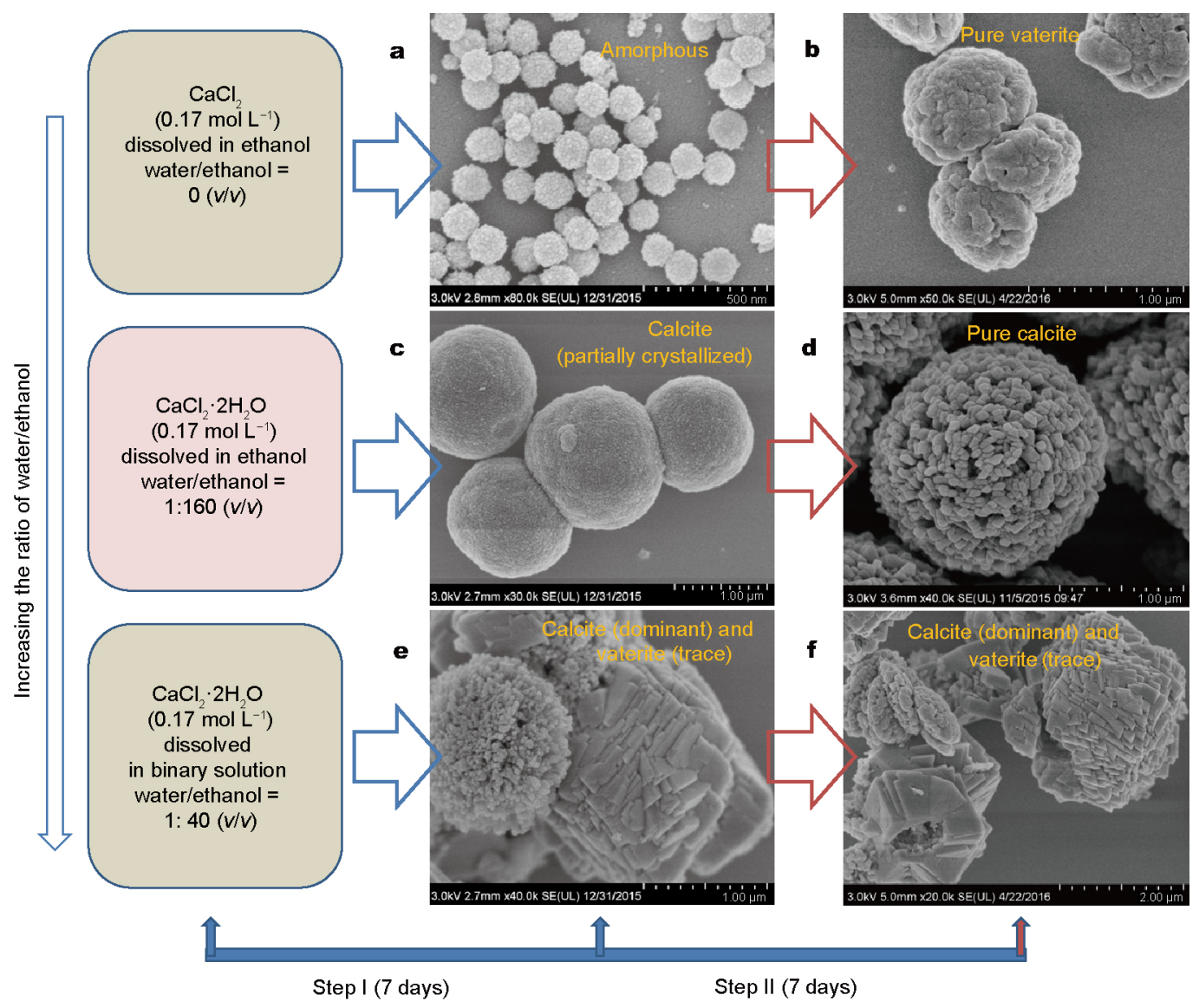

Figure 2 SEM images of samples obtained by two-step vapor-diffusion/aging crystallization strategy. (a, c, e) SEM images of the intermediate products synthesized in the first step: (a) $R=0$; (c) $R=1 / 160$; (e) $R=1 / 40$. (b, d, f) SEM images of the final products after two-step synthetic process: (b) $R=0$; (d) $R=1 / 160$; (f) $R=1 / 40$.

of morphologies were obtained in the first step (Fig. 2e, f): a majority of idiomorphic non-porous calcite rhombohedra crystals, and a minority of flower-like crystals that were confirmed as the vaterite phase by XRD characterization (Fig. S6c). The obtained polymorphs were similar to those of previously reported $\mathrm{CaCO}_{3}$ precipitations formed in absolute water [30], revealing that the ethanol solvent effect on the polymorph discrimination became weaker when the $R$-value was above $1 / 40$. Meanwhile, we noted that the vaterite in the mixture continued to readily convert into calcite during the aging process in the second step, which was confirmed by the significant increase in the calcite/vaterite intensity ratio in XRD diffraction (Fig. S6d). This result was consistent with the prevalent vaterite-to-calcite transformation mechanism whereby vaterite, with a metastable phase and a high surface area, had the tendency to undergo a dissolution and recrystallization process to become the more stable calcite phase [14].

On the basis of the above results, we found that the polymorph and morphology of the $\mathrm{CaCO}_{3}$ precipitate could be controlled accurately by selecting specific water/ethanol ratios in the binary solution via a $\mathrm{CO}_{2}$ vapor-diffusion reaction. Meanwhile, the results indicated that the presence of a tiny proportion of water was favorable to induce a spherical/porous calcite polycrystalline structure. More importantly, we hypothesized that more particular structures, besides PCMSs, could be fabricated by further manipulation of the thermodynamics and kinetics of crystallization, based on the present additive-free synthetic strategy, to satisfy different requirements in biomedical applications.

\section{Antibacterial protein loading capacity of PCMSs}

The PCMSs are expected to be applied in gene and protein delivery because of their highly open, large pores and large surface area. In this study, IgY, possessing a high molecular weight of about $180 \mathrm{kDa}$ and efficient antibacterial activity [32], was employed as a model drug protein to investigate the encapsulation efficiency of PCMS. To load IgY into PCMSs, the PCMSs powders were mixed with deionized 
water solution containing IgY protein $\left(1 \mathrm{mg} \mathrm{mL}^{-1}\right)$ through a gentle stirring process at $4^{\circ} \mathrm{C}$. Meanwhile, a typical rhombohedral calcite microcrystal (denoted as CMCs; the morphology of CMCs is shown in Fig. S7) was used as counterpart sample to determine the effect of the pore structure on the drug loading capacity. As shown in Fig. 3a, the FTIR spectrum of IgY loaded PCMSs (PCMSs-IgY) shows a specific absorption peak at $1648 \mathrm{~cm}^{-1}$, originating from IgY molecules, which confirms that IgY had been loaded successfully into the PCMSs. The IgY loading efficiency of PCMSs was measured to be about $31 \%$, which was much higher than that of CMCs (about 9.4\%). Meanwhile, their loading capacities, defined as the total loading of IgY in per unit mass of IgY loaded $\mathrm{CaCO}_{3}$, were calculated as approximately $72.0 \mathrm{mgg}^{-1}$ and $23.0 \mathrm{mgg}^{-1}$ for PCMSs and CMCs, respectively (Fig. $3 \mathrm{~b}$ ). The above results demonstrated that the PCMSs are beneficial for high-capacity protein adsorption, probably because of their unique large surface and open structure, which provide plenty of active sites and physical space for protein adsorption. Furthermore, we studied the IgY release behavior from PCMSs. As shown in Fig. S8, the accumulated release proportion of IgY is about $40.9 \%$ in $1 \mathrm{~h}$, and $82.9 \%$ in $8 \mathrm{~h}$. Based on this result, we believed that IgY could release relatively slowly from PCMS in actual oral environment and thus retain their therapeutic value over an extended period compared with the free IgY formulation.

\section{Antibacterial performance of PCMSs-IgY}

Furthermore, to evaluate the antibacterial performance of the PCMSs-IgY, S. mutans, which generally causes severe tooth demineralization and cavity formation, was chosen as the representative bacterial model in this study $[33,34]$. The inhibitory effect of three samples (PCMSs-IgY, pure
PCMSs, and free IgY; the IgY concentration was fixed at $4.5 \mathrm{mg} \mathrm{mL}^{-1}$ for IgY-containing samples) were examined against $S$. mutans, based on the colony counting approach (Fig. 4a). Fig. 4b shows typical images of the agar plates onto which a certain volume of $S$. mutans medium was re-cultivated after co-incubation with each sample for $12 \mathrm{~h}$. To compare the inhibitory effect between each treatment quantitatively, the corresponding CFU on the agar plate was counted, as shown in Fig. 4c. The results showed that pure PCMSs had a negligible antibacterial effect on $S$. mutans. By contrast, the surviving $S$. mutans counts of two IgY-containing samples (free IgY and PCMSs-IgY) showed a log reduction of 4.8 and 5.3, respectively, compared with that of the control group. More importantly, the inhibitory effect of PCMSs-IgY was higher than that of the free IgY formulation. This revealed the positive influence of PCMSs as antibacterial protein carriers, which was mostly attributed to the sustained release behavior of the IgY protein. Hence, the obtained results indicated that PCMSs-IgY could inhibit the growth of oral bacteria efficiently based on an antibacterial protein delivery strategy.

\section{High performance in remineralization and occluding of exposed dentin}

Recently, efforts have been made to construct novel $\mathrm{CaCO}_{3}$ materials possessing high-performance bone bioactivity, because of increasing interest in the fields of bone implantation and dental treatment [35]. In this study, the PCMSs were immersed into a PBS solution $(1 \times)$ at $37^{\circ} \mathrm{C}$ and collected at different time intervals ( 2 and $24 \mathrm{~h}$ ) to measure the rate of apatite formation. Meanwhile, the solid CMC, as mentioned above, was also employed as a reference sample to compare the effect of the unique porous structure on the mineralization process of $\mathrm{CaCO}_{3}$.
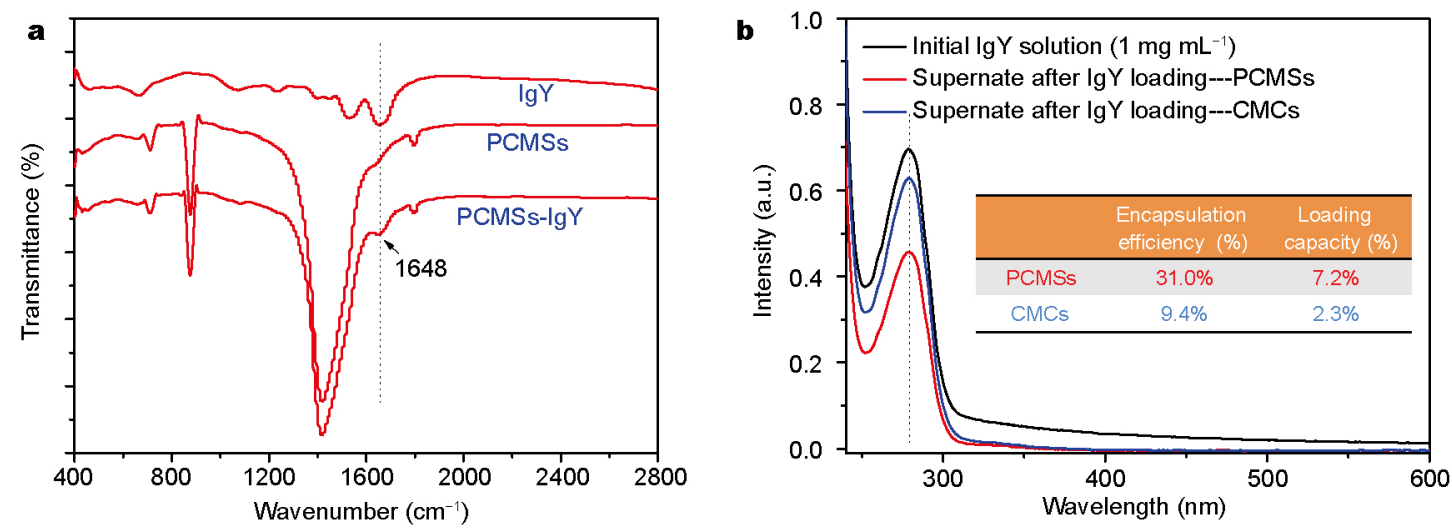

Figure 3 Characterizations of IgY protein loading capacity for PCMSs. (a) FTIR spectra of free IgY, empty PCMSs and PCMSs-IgY. The arrow indicates the specific absorption peak at $1648 \mathrm{~cm}^{-1}$. (b) UV-vis spectra of supernatant solution after loading PCMSs and CMCs with the same initial concentration of $\operatorname{IgY}\left(1 \mathrm{mg} \mathrm{mL}^{-1}\right)$, separately. The inside table shows the calculated encapsulation efficiency and loading capacity of each sample. 

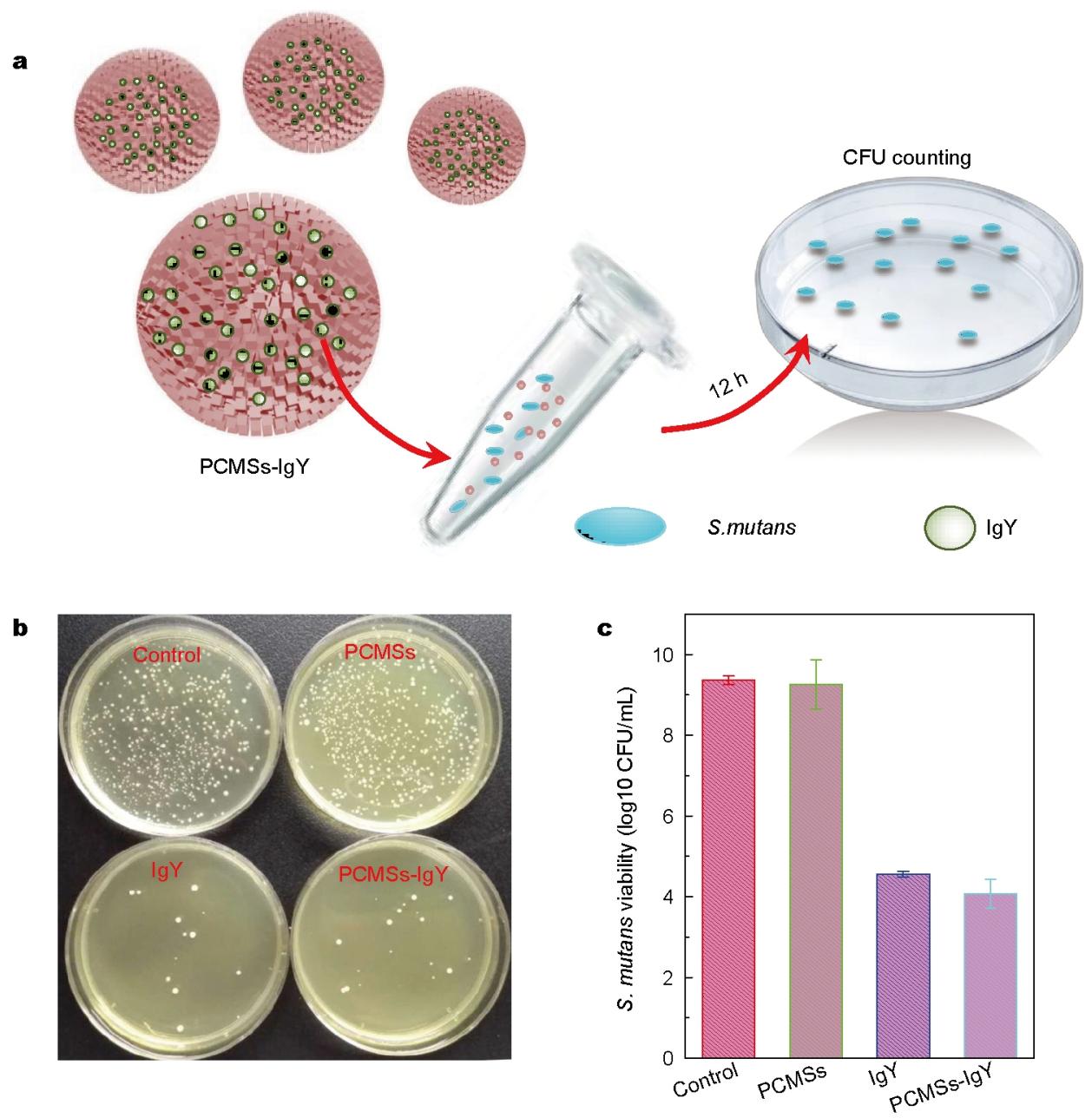

Figure 4 (a) Schematic demonstration of the in vitro antibacterial activity evaluation of the PCMSs-IgY against S. mutans. (b, c) The S. mutans colonies (b) and the corresponding surviving S. mutans counts (c) after different treatments for $12 \mathrm{~h}$.

Interestingly, a significant change in surface morphology to lath-like aggregations was observed for PCMSs after being immersed in PBS for only $2 \mathrm{~h}$ (Fig. $5 \mathrm{a}$ and Fig. S9), which was markedly different from their high-stability performance in deionized water. Furthermore, the crystal structure change of the PCMSs after incubation was investigated qualitatively using Raman spectroscopy (Fig. 5b and Fig. S10). As can be seen from Fig. 5b, the characteristic peaks of calcite at $156,282,713$, and $1087 \mathrm{~cm}^{-1}$ for PCMSs [36] were observed after $2 \mathrm{~h}$ of incubation. Instead, strong peaks for $\mathrm{P}-\mathrm{O}$ symmetric bending vibration at about $431 \mathrm{~cm}^{-1}$, O-P-O antisymmetric bending vibration at about $591 \mathrm{~cm}^{-1}$, and $\mathrm{P}-\mathrm{O}$ symmetric stretching vibration at about $962 \mathrm{~cm}^{-1}$ were observed, which are ascribed to the formation of hydroxyapatite [37]. Thus, the above results confirmed a complete conversion of PCMSs to hydroxyapatite within $2 \mathrm{~h}$. In addition, the SEM image in Fig. $5 c$ shows negligible morphological change of the lath-like MPs after incubation for $24 \mathrm{~h}$, indicating that hydroxyapatite is the final product of the transformation route for PCMSs.

In contrast, for the reference CMCs sample, only a few lath-like agglomerates with a nanometer particle size were observed on the surface of the rhombohedra MPs after $2 \mathrm{~h}$ of incubation (Fig. $5 \mathrm{~d}$ and Fig. S11). Meanwhile, the Raman spectrum in Fig. 5e shows that the absorbance peaks for calcite and hydroxyapatite co-existed, with the major peaks for calcite being significantly stronger than those of hydroxyapatite, demonstrating that only a small proportion of the $\mathrm{CaCO}_{3}$ on the particle surface was converted to apatite within $2 \mathrm{~h}$. Furthermore, after incubation for $24 \mathrm{~h}$, the characteristic peaks of calcite seem to disappear in the Raman spectra (as seen in Fig. S12); however, the existence of a smooth surface morphology in certain 

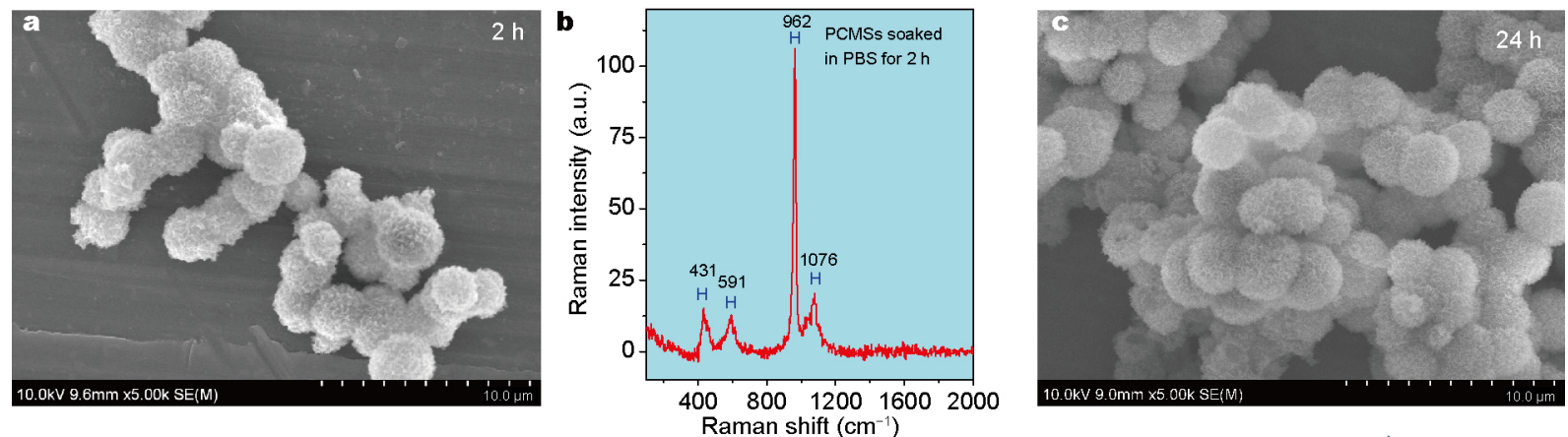

Raman shift $\left(\mathrm{cm}^{-1}\right)$
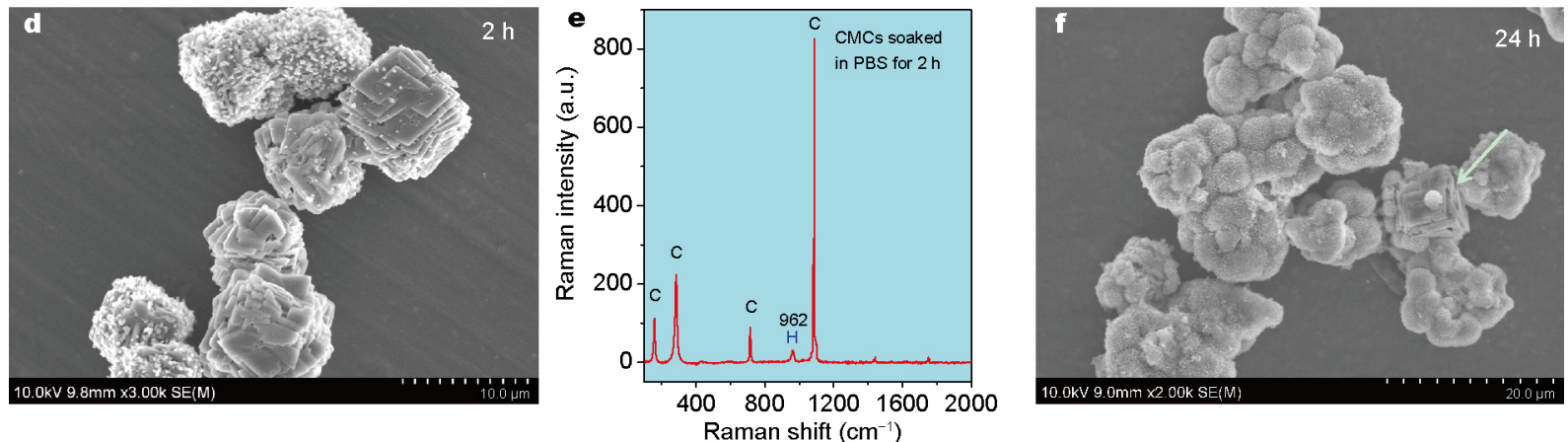

Figure 5 SEM images and Raman spectra showing the conversion of samples to hydroxyapatite in PBS solution. (a, c) SEM images of PCMSs after soaking in PBS for (a) 2 and (c) 24h. Lath-like surface morphology formed after soaking for 2h. (b) Raman spectra of PCMSs after soaking in PBS for $2 \mathrm{~h}$. $\mathrm{H}$ indicate the characteristic peaks of hydroxyapatite. (d, f) SEM images of counterpart samples CMCs after soaking in PBS for (a) 2 and (c) $24 \mathrm{~h}$. Nanometer sized lath-like agglomerates are shown on the surface of rhombohedral calcite after soaking CMCs in PBS for $2 \mathrm{~h}$. The arrow in Fig. $5 \mathrm{f}$ indicates the unreacted rhombohedral calcite. (e) Raman spectrum of CMCs after soaking in PBS for $2 \mathrm{~h}$. C indicates the characteristic peaks of calcite. Only a minority of calcite converts to hydroxyapatite structure for CMCs within $2 \mathrm{~h}$.

place was observed on the SEM image (Fig. 5f), revealing that the transformation was not complete.

The above in vitro experimental results demonstrated that the PCMSs have higher reactivity for the transformation to hydroxyapatite in PBS compared with conventional CMCs. The porous structure could provide a high-volume reservoir for the adsorbed phosphate ions, thus accelerating the generation of a local ions supersaturation condition for the precipitation of hydroxyapatite crystals [38]. Such rapid transformation to apatite could also be achieved when immersing PCMSs in other biological media, such as the filtrated human saliva (Fig. S13). Therefore, we hypothesize that rapid remineralization of PCMSs will be feasible under various biological conditions.

Dental hypersensitivity is an acute dental disease that generally occurs when the tubules open at the dentin surface and become exposed to the oral environment [39]. Among various desensitizing approaches, occluding the dentinal tubules by depositing dentin-biomimetic minerals into the dentinal tubules, has received most attention owing to its rapid and noninvasive nature [4,40-42]. The rapid transformation of PCMSs to bone minerals has been confirmed under different conditions in this study; therefore, it is anticipated that this newly-designed material could exhibit high performance in treating sensitive teeth. To evaluate the effectiveness of PCMSs on dentin tubule occlusion, a simple in vitro study was conducted by brushing the dentin surface with the PCMSs in glycerinum. Meanwhile, the most used $\mathrm{CaCO}_{3}$ in current commercial toothpaste (MCCPs) was also employed for comparison. As shown in Fig. 6a, the dentin tubules were completely open in the untreated dentin specimen. In contrast, a considerably high density of porous products within the tubules was observed (Fig. 6b and Fig. S14) after the dentin specimen received sequential treatments by brushing the dentin surface for $2 \mathrm{~min}$ and incubation in PBS for $15 \mathrm{~min}$. The superstructures of the porous particles and dentin could be distinguished clearly when viewing the specimen under high magnification (Fig. 6c). Meanwhile, we also observed the structural collapse of spherical MPs, demonstrating that the remineralization of PCMSs occurred upon rinsing in PBS. Importantly, Fig. 6d, e and 


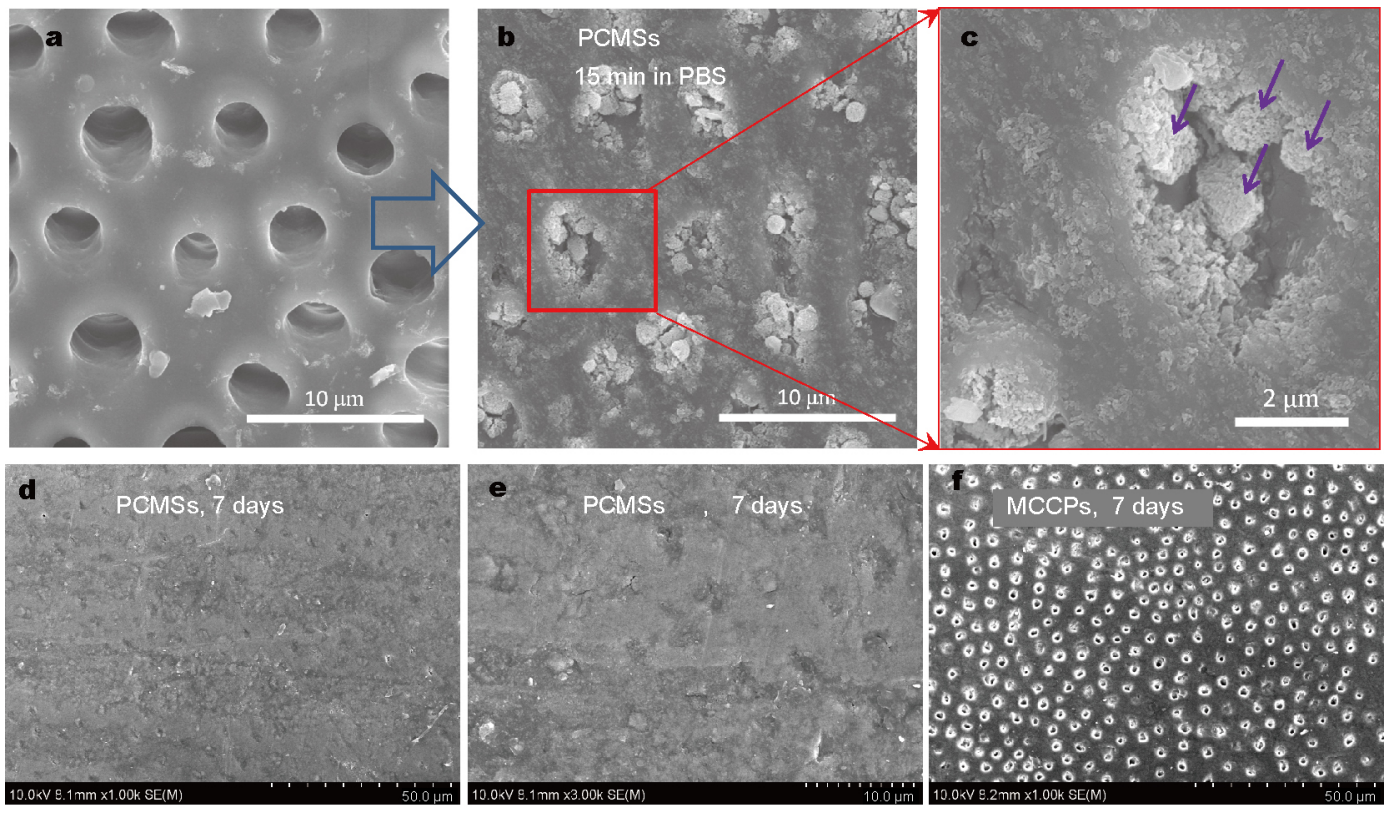

$\mathbf{g}$

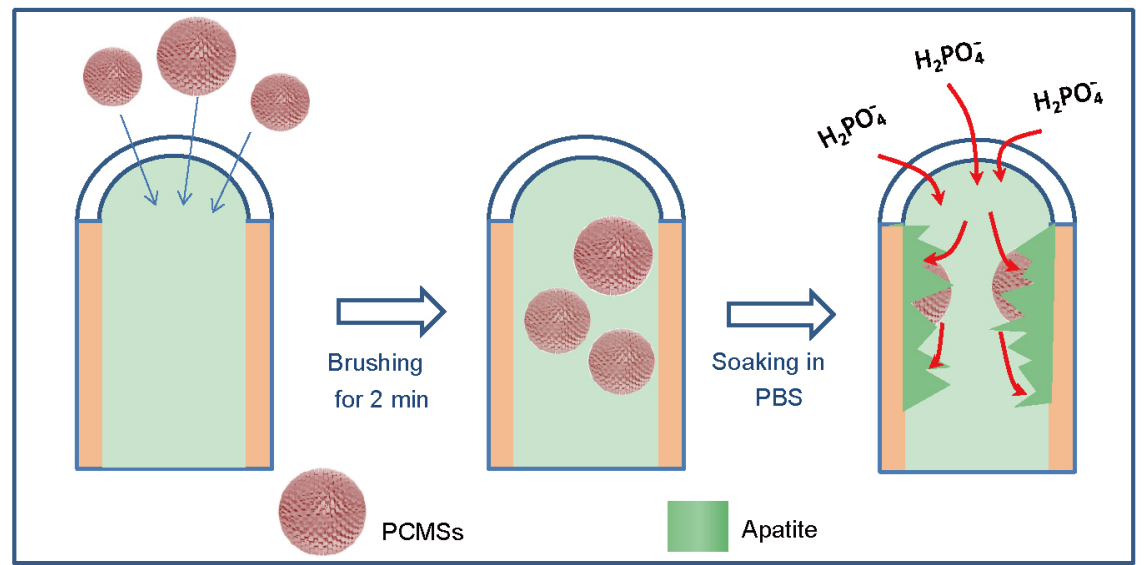

Figure 6 SEM images of dentinal tubules before (a) and after treatment with the PCMSs (b-e) and MCCPs group (f). (b, c) The PCMSs-treated dentin surface was incubated in PBS solution for $15 \mathrm{~min}$. The arrows in Fig. $6 \mathrm{c}$ indicate the broken porous particles within tubules. (d-f) SEM images of the groups containing PCMSs (d, e) and MCCPs (f) after tooth-brushing for 7 days: (d, f) magnified $\times 1000$, (e) magnified $\times 3000$. (g) Schematic illustration of the hydroxyapatite formation mechanism of PCMSs within dentin tubules.

Fig. S15 show that the tubules were completely occluded after repeatedly brushing with PCMSs twice a day for 7 days. Meanwhile, as seen in Fig. S16, the cross sectional view of the teeth in the PCMSs group shows that part of the tubules contains a crystalline precipitate. However, for the teeth in the MCCPs group, the occlusion of the open dentinal tubules was insufficient (Fig. $6 \mathrm{f}$ and Fig. S17). The above results confirm that PCMSs are highly efficient for occluding open dentinal tubules.

Fig. $6 \mathrm{~g}$ illustrates the possible formation mechanism of the minerals within dentinal tubules after treatment with PCMSs. PCMSs are smaller than common dentinal tubules; therefore, they can penetrate easily into the tubules by mechanical brushing. Afterwards, the phosphate ions in the PBS buffer permeate into the tubules and react rapidly with embedded PCMSs to form apatite crystals under the same transformation mechanism as that of the non-dentin containing system mentioned above. Collectively, these findings suggest that PCMSs can not only inhibit demineralization efficiently based on an antibacterial protein delivery strategy, but also can enhance remineralization of damaged teeth as a fast-reacting biomaterial.

\section{CONCLUSIONS}

In summary, newly designed calcite microspheres, featuring a symmetry-breaking assembled porous structure, 
were prepared successfully using a two-step vapor-diffusion/aging crystallization approach. The building blocks of PCMSs are the high-stability calcite phase, and their morphological characteristics, including a spherical shape and porous structure, were maintained well in deionized water for more than three months, without the need of any foreign stabilizer. Meanwhile, the PCMSs exhibited high adsorption capability for IgY, leading to an enhanced antibacterial effect against oral bacteria S. mutans. Significantly, the results demonstrated that the PCMSs possessed rapid remineralization ability, and could be fully converted into bone minerals within $2 \mathrm{~h}$ when soaked in physiological media. The PCMSs could also occlude dentinal tubules efficiently, confirming their powerful potential as catalysts in the repair of dental hard tissue. Therefore, the developed PCMSs possess multiple advantages in terms of their preparation strategy, structural properties, and medical functionalities, which show great potential in the repair of dental hard tissue.

\section{Received 10 March 2017; accepted 19 April 2017;} published online 16 May 2017

1 Trushina DB, Bukreeva TV, Kovalchuk MV, et al. $\mathrm{CaCO}_{3}$ vaterite microparticles for biomedical and personal care applications. Mater Sci Eng-C, 2014, 45: 644-658

2 Cury JA, Simões GS, Del Bel Cury AA, et al. Effect of a calcium carbonate-based dentifrice on in situ enamel remineralization. Caries Res, 2005, 39: 255-257

3 Elabbadi A, Jeckelmann N, Haefliger O, et al. Selective coprecipitation of polyphenols in bioactive/inorganic complexes. ACS Appl Mater Interfaces, 2011, 3: 2764-2771

4 Lu P, Arai K, Kuboyama N. Possibility of application of calcium carbonate in pulpotomy of rat molars. Pediatric Dental J, 2010, 20: 45-56

5 Pickles MJ, Evans M, Philpotts CJ, et al. In vitro efficacy of a whitening toothpaste containing calcium carbonate and perlite. Int Dental J, 2005, 55: 197-202

6 Ueno Y, Futagawa H, Takagi Y, et al. Drug-incorporating calcium carbonate nanoparticles for a new delivery system. J Control Release, 2005, 103: 93-98

7 Wei W, Ma GH, Hu G, et al. Preparation of hierarchical hollow $\mathrm{CaCO}_{3}$ particles and the application as anticancer drug carrier. $\mathrm{J}$ Am Chem Soc, 2008, 130: 15808-15810

8 Kim YY, Schenk AS, Ihli J, et al. A critical analysis of calcium carbonate mesocrystals. Nat Commun, 2014, 5: 4341

9 Yu JG, Guo H, Davis SA, et al. Fabrication of hollow inorganic microspheres by chemically induced self-transformation. Adv Funct Mater, 2006, 16: 2035-2041

10 Lee K, Wagermaier W, Masic A, et al. Self-assembly of amorphous calcium carbonate microlens arrays. Nat Commun, 2012, 3: 725

11 Wolf SE, Leiterer J, Pipich V, et al. Strong stabilization of amorphous calcium carbonate emulsion by ovalbumin: gaining insight into the mechanism of 'polymer-induced liquid precursor' processes. J Am Chem Soc, 2011, 133: 12642-12649

12 Qi C, Zhu YJ, Lu BQ, et al. ATP-stabilized amorphous calcium carbonate nanospheres and their application in protein adsorption.
Small, 2014, 10: 2047-2056

13 Hetherington NBJ, Kulak AN, Kim YY, et al. Porous single crystals of calcite from colloidal crystal templates: ACC is not required for nanoscale templating. Adv Funct Mater, 2011, 21: 948-954

14 Won YH, Jang HS, Chung DW, et al. Multifunctional calcium carbonate microparticles: synthesis and biological applications. J Mater Chem, 2010, 20: 7728-7733

15 Zhang J, Li Y, Xie H, et al. Calcium carbonate nanoplate assemblies with directed high-energy facets: additive-free synthesis, high drug loading, and sustainable releasing. ACS Appl Mater Interfaces, 2015, 7: 15686-15691

16 Volodkin DV, Petrov AI, Prevot M, et al. Matrix polyelectrolyte microcapsules: new system for macromolecule encapsulation. Langmuir, 2004, 20: 3398-3406

17 Wang X, Kong R, Pan X, et al. Role of ovalbumin in the stabilization of metastable vaterite in calcium carbonate biomineralization. J Phys Chem B, 2009, 113: 8975-8982

18 Zhang Z, Gao D, Zhao H, et al. Biomimetic assembly of polypeptide-stabilized $\mathrm{CaCO}_{3}$ nanoparticles. J Phys Chem B, 2006, 110: 8613-8618

19 Cölfen H, Antonietti M. Crystal design of calcium carbonate microparticles using double-hydrophilic block copolymers. Langmuir, 1998, 14: 582-589

20 Chen SF, Yu SH, Jiang J, et al. Polymorph discrimination of $\mathrm{CaCO}_{3}$ mineral in an ethanol/water solution: formation of complex vaterite superstructures and aragonite rods. Chem Mater, 2006, 18: $115-122$

21 de Leeuw NH, Parker SC. Surface structure and morphology of calcium carbonate polymorphs calcite, aragonite, and vaterite: an atomistic approach. J Phys Chem B, 1998, 102: 2914-2922

22 Montes-Hernandez G, Renard F, Findling N, et al. Formation of porous calcite mesocrystals from $\mathrm{CO}_{2}-\mathrm{H}_{2} \mathrm{O}-\mathrm{Ca}(\mathrm{OH})_{2}$ slurry in the presence of common domestic drinks. CrystEngComm, 2015, 17: 5725-5733

23 Wang J, Li D, Li T, et al. Gelatin tight-coated poly(lactide-co-glycolide) scaffold incorporating rhBMP-2 for bone tissue engineering. Materials, 2015, 8: 1009-1026

24 Zhang N, Wang Y, Xu W, et al. Poly(lactide-co-glycolide)/hydroxyapatite porous scaffold with microchannels for bone regeneration. Polymers, 2016, 8: 218

25 Domingo C, Loste E, Gómez-Morales J, et al. Calcite precipitation by a high-pressure $\mathrm{CO}_{2}$ carbonation route. J Supercritical Fluids, 2006, 36: 202-215

26 Lee HS, Ha TH, Kim K. Fabrication of unusually stable amorphous calcium carbonate in an ethanol medium. Mater Chem Phys, 2005, 93: $376-382$

27 Saleem IY, Vordermeier M, Barralet JE, et al. Improving peptidebased assays to differentiate between vaccination and mycobacterium bovis infection in cattle using nanoparticle carriers for adsorbed antigens. J Control Release, 2005, 102: 551-561

28 Ikoma T, Tonegawa T, Watanaba H, et al. Drug-supported microparticles of calcium carbonate nanocrystals and its covering with hydroxyapatite. J Nanosci Nanotech, 2007, 7: 822-827

29 Cai WY, Feng LD, Liu SH, et al. Hemoglobin-CdTe- $\mathrm{CaCO}_{3} @$ polyelectrolytes 3D architecture: fabrication, characterization, and application in biosensing. Adv Funct Mater, 2008, 18: 3127-3136

30 Sand KK, Rodriguez-Blanco JD, Makovicky E, et al. Crystallization of $\mathrm{CaCO}_{3}$ in water-alcohol mixtures: spherulitic growth, polymorph stabilization, and morphology change. Cryst Growth Des, 2012, 12: 842-853

31 Wang T, Cölfen H, Antonietti M. Nonclassical crystallization: mesocrystals and morphology change of $\mathrm{CaCO}_{3}$ crystals in the 
presence of a polyelectrolyte additive. J Am Chem Soc, 2005, 127: 3246-3247

32 Smith DJ, King WF, Godiska R. Passive transfer of immunoglobulin $\mathrm{Y}$ antibody to Streptococcus mutans glucan binding protein $\mathrm{B}$ can confer protection against experimental dental caries. Infection Immun, 2001, 69: 3135-3142

33 Nilsson E, Kollberg H, Johannesson M, et al. More than 10 years' continuous oral treatment with specific immunoglobulin $\mathrm{Y}$ for the prevention of Pseudomonas aeruginosa infections: a case report. J Medicinal Food, 2007, 10: 375-378

34 Klein MI, Hwang G, Santos PHS, et al. Streptococcus mutans-derived extracellular matrix in cariogenic oral biofilms. Front Cell Infect Microbiol, 2015, 5: 10

35 Kim S, Park CB. Mussel-inspired transformation of $\mathrm{CaCO}_{3}$ to bone minerals. Biomaterials, 2010, 31: 6628-6634

36 de La Pierre M, Carteret C, Maschio L, et al. The Raman spectrum of $\mathrm{CaCO}_{3}$ polymorphs calcite and aragonite: a combined experimental and computational study. J Chem Phys, 2014, 140: 164509

37 Yamini D, Devanand Venkatasubbu G, Kumar J, et al. Raman scattering studies on PEG functionalized hydroxyapatite nanoparticles. Spectrochimica Acta Part A-Mol Biomolecular Spectroscopy, 2014, 117: 299-303

38 Chiang YC, Lin HP, Chang HH, et al. A mesoporous silica biomaterial for dental biomimetic crystallization. ACS Nano, 2014, 8: 12502-12513

39 Absi EG, Addy M, Adams D. Dentine hypersensitivity. A study of the patency of dentinal tubules in sensitive and non-sensitive cervical dentine. J Clin Periodontol, 1987, 14: 280-284
40 Oh DX, Prajatelistia E, Ju SW, et al. A rapid, efficient, and facile solution for dental hypersensitivity: the tannin-iron complex. Sci Rep, 2015, 5: 10884

41 Tsai WS, Placa SJ, Panagakos PS. Clinical evaluation of an in-office desensitizing paste containing $8 \%$ arginine and calcium carbonate for relief of dentin hypersensitivity prior to dental prophylaxis. Am J Dent, 2012, 25: 165-170

42 Suge T, Ishikawa K, Kawasaki A, et al. Duration of dentinal tubule occlusion formed by calcium phosphate precipitation method: in vitro evaluation using synthetic saliva. J Dental Res, 1995, 74: 1709-1714

Acknowledgments This work was supported by the National Natural Science Foundation of China (51402329 and 81500806), the Science Foundation for Youth Scholar of State Key Laboratory of High Performance Ceramics and Superfine Microstructures (SKL201404) and Shanghai Excellent Academic Leaders Program (14XD1403800).

Author contributions $\mathrm{Ma} \mathrm{M}$ and Yan $\mathrm{Y}$ contributed to this work equally. Ma M designed and synthesized the samples; Ma M, Yan Y, Chern S, Qi S and Shang G performed the experiments; Ma M wrote the paper with support from Qi C, Chen $\mathrm{H}$ and Wang R. All authors contributed to the general discussion.

Conflict of interest The authors declare that they have no conflict of interest.

Supplementary information Supporting data are available in the online version of the paper. 

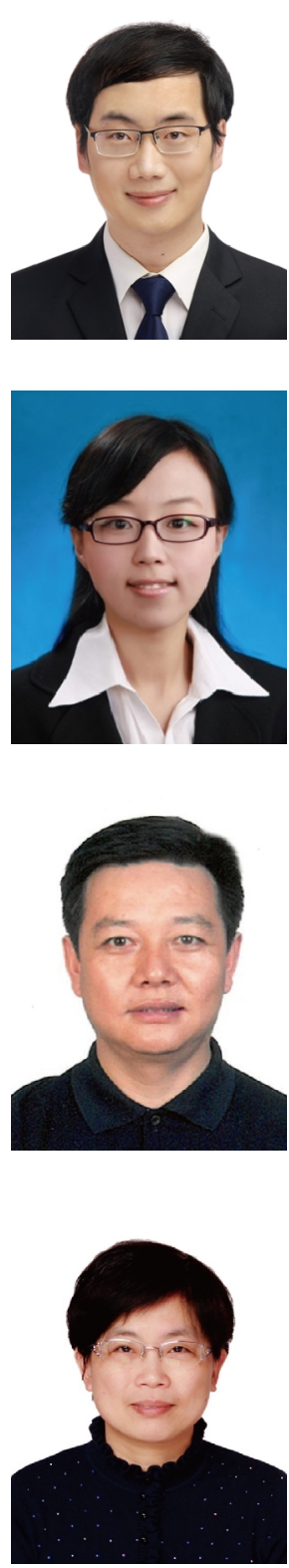

Ming Ma received his PhD degree from Shanghai Institute of Ceramics, Chinese Academy of Sciences (SICCAS) in 2013. $\mathrm{He}$ is now an associate professor of SICCAS. His research focuses on the design of mesoporous materials for biomedical applications including anticancer drug delivery, medical imaging and dental restoration.
Yanhong Yan obtained her PhD degree from Wuhan University under the supervision of Prof. Mingwen Fan. She is now a dentist at the Department of Pediatric Dentistry, School of Stomatology, Tongji University. Her research focuses on the structure and properties of anti-dental caries biomaterials.
Raorao Wang obtained his $\mathrm{PhD}$ degree from Matsumoto Dental University in Japan under the supervision of Prof. Hiroo Miyazawa. He is now the director of Stomatological Department, Shanghai Tenth People's Hospital of Tongji University. His research interest focuses on the stem cell-based biological tooth repair and regeneration.
Hangrong Chen received her $\mathrm{PhD}$ degree from SICCAS in 2001. She is now a professor of SICCAS, and deputy director of the State Key Laboratory of High Performance Ceramics and Superfine Microstructure. Her research areas include the synthesis of mesoporous materials, multifunctional inorganic biomedical nanomaterials and environmental catalytic materials.

\section{多孔方解石相碳酸钻微球的自组装合成及口腔修复应用}

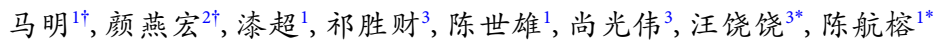

摘要 多孔碳酸钙微球的弱水溶液稳定性和低再矿化速率限制了其在生物医药领域的应用. 为了解决该问题, 本论文通过“气体扩散-陈化” 两步策略制备了具有大孔结构和方解石相的碳酸钙微球材料. 该合成策略反应条件温和, 同时不需要使用模板剂和晶型导向剂, 能够确 保微球产物的生物安全性. 所制备的碳酸钲微球可以吸附大量的免疫球蛋白 IgY, 并表现出增强抑制口腔变形链球菌的能力. 此外, 研究显 示该微球可以在水溶液中保持较高的结构稳定性, 而在人体体液和唾液环境中快速再矿化并转变成羟基磷灰石结构, 对牙本质小管具有 较佳的封闭效果, 为其用于治疗牙本质过敏症提供了依据. 